\title{
Applications of Bubblegram Imaging
}

\author{
Weimin $\mathrm{Wu}^{1}$, Naiqian $\mathrm{Cheng}^{1}$, Alasdair C. Steven ${ }^{1}$ and Juan Fontana ${ }^{2}$. \\ ${ }^{1 .}$ Laboratory of Structural Biology Research, National Institute of Arthritis, Musculoskeletal \\ and Skin Diseases, National Institutes of Health, Bethesda, MD, U.S.A. \\ 2. Astbury Centre for Structural Molecular Biology, University of Leeds, U.K.
}

Dense packing of macromolecules in cellular compartments and higher-order assemblies makes it difficult to pick out even quite large components in electron micrographs, despite nominally high resolution. Immunogold labeling and histochemical procedures offer ways to map certain components but are limited in their applicability. This presentation will review our progress to date with an alternative procedure that we call "Bubblegram Imaging". It is based on the principle that some proteins are acutely sensitive to radiation damage in the form of bubbling, especially when deeply embedded in nucleic acid, and that property may be exploited to localize them. Our first application was in determining the "inner body" structure of bacteriophage phiKZ (1; Fig. 1A). Subsequent applications have targeted, among other specimens, herpes simplex virus capsid (2; Fig. 1B), bacteriophage P22 (3; Fig. 1C). human immunodeficiency virus (4; Fig. 1D), and bacteriophage T7 (5; Fig. 1E),

We have used a CM200-FEG electron microscope (FEI) with a Gatan 626 cryo-holder maintaining a specimen temperature of $\sim-180^{\circ} \mathrm{C}$, operated at $120 \mathrm{keV}$ and 38,000x magnification to record dose series. Each 1-sec exposure corresponded to $\sim 15 \mathrm{e}-/ \AA^{2}$. Exposures were, typically, $10 \mathrm{sec}$ apart. Micrographs were recorded on film, digitized, and the data were processed using Bsoft and EMAN2. Micrographs in a given dose series were aligned to the first (low-dose) image. Particles' orientations were determined from first exposure images and also used for subsequent images at progressively higher doses.

Whereas individual proteins in these viruses are essentially impossible to detect in conventional lowdose images, the strong contrast between bubbles and the surrounding material enables their detection. With phiKZ, the bubbling principle is the inner body proteins; in T7, it is the core proteins gp14, gp15, and gp16; in the HSV nucleocapsid, it is mostly the viral protease underlying the five-fold vertices; in $\mathrm{HIV}$, it is the nucleocapsid (NC) protein. However, the bubblegram patterns tend to be stochastically variable from particle to particle, but emerge reproducibly after combining data in a 3D density map. We refer to the resulting low-density region as the "gas cloud". In tailed phages like T7, the portal axis and the unique vertex through which it passes may be readily identified and afford a reference point. In phiKZ, the inner body proteins are not aligned with portal axis and required an alternative computational approach (1). While the approach has considerable potential for future practical applications, it remains to be established why certain proteins happen to be particularly susceptible to bubbling [6].

References:

[1] W Wu et al, Science 335 (2012), p.182.

[2] W Wu et al, JVI 90 (2016), p.5176.

[3] W Wu et al, mBIO 7 (2016), e01152-16

[4] J Fontana et al, JVI 89 (2015), p.9765.

[5] N Cheng et al, J Struct Biol 385 (2014), p.250.

[6] This research was supported by the intramural research program of NIAMS. 

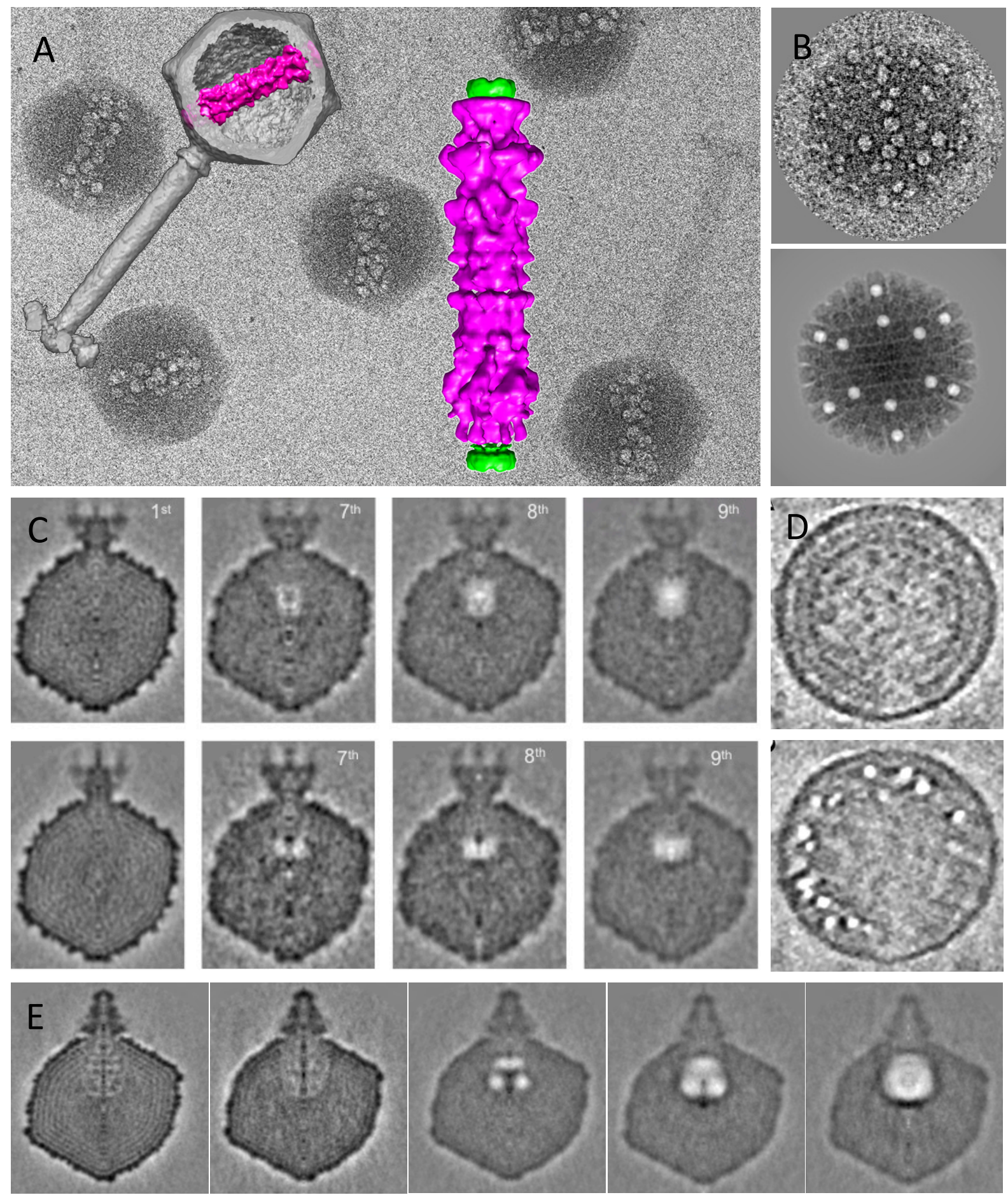

Figure 1. Applications of bubblegram imaging. (A) phiKZ bubblegram and reconstruction of inner body. (B) HSV nucleocapsid bubblegram (upper half) and simulation of bubbles at sub-vertex sites (bottom half). (C) P22 reconstructions from bubblegram images of $1^{\text {st }}, 7^{\text {th }}, 8^{\text {th }}$, and $9^{\text {th }}$ exposures of a 20 -minus mutant (upper half) and a 16-minus mutant (bottom half). (D) Tomographic slices through an HIV immature virion without bubbling (upper half) and with bubbling (bottom half). (E) T7 reconstructions of bubblegram images from the $1^{\text {st }}, 3^{\text {rd }}, 5^{\text {th }}, 7^{\text {th }}$, and $9^{\text {th }}$ exposures of a dose series (left to right). 